\title{
I fattori soggettivi nel «moderno capitalismo». La complicata ricezione italiana e le questioni insolute nel pensiero di W. Sombart
}

\author{
Vitantonio Gioia*
}

\section{Introduzione}

Werner Sombart ha sempre rappresentato «a significant intellectual puzzle» per i suoi interpreti ${ }^{1}$, a causa degli sviluppi contraddittori del suo pensiero. Nell'analisi delle contraddizioni di Sombart si è, spesso, fatto riferimento alla sua acuta sensibilità storica e al modo in cui egli, data la sua peculiare dimensione psicologica, reagì ai radicali mutamenti dei suoi tempi. Talvolta, si è enfatizzato il ruolo determinante delle sue scelte ideologiche o di sue presunte vocazioni opportunistiche. Ovviamente, sono aspetti che, nella lettura complessiva dell'opera sombartiana, non vanno trascurati. Tuttavia mi sembra che spesso il ricorso a tali temi abbia finito con l'assumere un peso così rilevante da sminuire il senso della complessità del tentativo scientifico di Sombart e dell' insieme delle questioni poste dal suo programma di ricerca.

In questo contributo mi soffermerò soprattutto sull'ipotesi che i risultati contraddittori dell'opera di Sombart siano da ricercare nel suo approccio epistemologico e nella peculiare configurazione dell'oggetto di ricerca che egli adottò. Questo consente, probabilmente, di valutare in modo diverso il tema

* Università del Salento, Dipartimento di Storia, Società, Studi sull'Uomo.

1 N. Stehr, R. Grundmann, Why Is Werner Sombart Not Part of the Core of Classical Sociology? From Fame to (Near) Oblivion, «Journal of Classical Sociology», 1 (2), 2001, p. 258. 
dell'influenza decisiva che K. Marx ha esercitato su Sombart (al di là delle critiche che egli rivolge al padre del 'socialismo scientifico'). Secondo Marx il capitalismo, promuovendo lo sviluppo delle forze produttive creava - seppure in maniera contraddittoria - le condizioni oggettive (organizzative e produttive) e soggettive (politiche e culturali) per la realizzazione del socialismo. In tal modo, si sarebbero realizzati quei processi di emancipazione umana e di progresso sociale, che rappresentavano la componente più importante dell'eredità illuministica nella cultura europea (eredità che proprio Marx aveva contribuito a liberare dalla 'sterile' dimensione utopica). Per molto tempo Sombart ha condiviso questa convinzione, divenuta una componente rilevante del suo programma di ricerca. La successiva percezione del carattere a-scientifico (e non realistico) di questa visione ha avuto effetti significativi sia con riferimento al tema del rapporto tra capitalismo e socialismo; sia con riferimento all'analisi del ruolo dei fattori del mutamento sociale.

La riflessione su questi aspetti sarà sviluppata in quattro sezioni:

- nella prima sezione cercherò, seppure molto rapidamente, di liberare la riflessione su Sombart da tre temi (il rapporto Sombart-Marx; il suo presunto razzismo e il suo rapporto con il nazismo), che talvolta sono stati enfatizzati al punto da distorcere l'interpretazione della sua opera;

- nella seconda ricostruirò gli elementi specifici del programma di ricerca di Sombart, con riferimento al tema trattato: i caratteri distintivi del capitalismo e il ruolo dei fattori soggettivi nella spiegazione del mutamento sociale;

- nella terza e quarta sezione mi soffermerò sulla recezione italiana dell'opera di Sombart con riferimento alla genesi e sviluppo del capitalismo italiano e con riferimento al rapporto tra sviluppo capitalistico, progresso sociale e crescita dei fattori soggettivi.

\section{Sombart, Marx, gli ebrei e il nazismo}

Sombart ha, per sua stessa ammissione, considerato il programma scientifico di Marx come un suo essenziale modello di riferimento. Questo, ovviamente, non autorizza a considerarlo un marxista. In effetti, se si considera il contesto scientifico entro cui egli si muoveva, quello della scuola storica tedesca dell'economia (da ora in poi GHS), non si farà fatica a riconoscere nella sua opera una posizione ampiamente condivisa (pur con qualche differenza) da autori come Schmoller, Max Weber, Arthur Spiethoff, J.A. Schumpeter (tanto per citarne alcuni). Proprio Schmoller aveva reso Marx e la sua opera come un oggetto accademicamente rilevante, poiché - al di là del giudizio negativo sulla sua proposta politica - ne aveva apprezzato le innovazioni teoriche: il modo in cui egli aveva saputo emanciparsi sia dal determinismo economicistico della scuola classica ${ }^{2}$, che dalle «esagerazioni dell'indirizzo idealistico» nel campo delle scienze so- 
ciali ${ }^{3}$. L'impianto materialistico di Marx aveva introdotto, in maniera scientificamente rigorosa, «il grande pensiero dell'evoluzione nelle scienze sociali», fondando «la ragione storica» delle «epoche economiche» e delle loro «differenze», contribuendo a far luce sui «lati oscuri della vita sociale $»^{4}$. Tuttavia, «il pensiero in sé giusto [è] stato da Marx, e ancor più dai suoi successori, esagerato», facendo dipendere meccanicamente «il processo della vita sociale, spirituale e politica dei popoli dalla produzione e riproduzione dei beni materiali» ${ }^{5}$. Questo ha portato alla sottovalutazione del ruolo dei fattori soggettivi nei processi di mutamento sociale e al disprezzo per la funzione svolta, in tal senso, dai sistemi politico-istituzionali ${ }^{6}$.

Il giovane Sombart riprende questi temi e, pur enfatizzando - contro Schmoller $-\ll$ il carattere puramente teorico» dell'approccio marxiano, non manca di sottolineare i limiti del materialismo storico, che egli individua nella carenza di un'appropriata fondazione psicologica nell'analisi economica e sociologica: il suo $<$ radicale oggettivismo (extremer Objektivismus) $\rangle^{7}$ trascurava $\ll$ l'approccio soggettivo (subjective Richtung) », non cogliendo il fatto che i fenomeni economici sono determinati, «in ultima analisi, dalla psiche del soggetto economico ${ }^{8}$. Ritorneremo su questo tema successivamente, ma certo il fatto che Sombart assuma Marx come un essenziale riferimento per la sua analisi del capitalismo moderno e si differenzi parzialmente da Schmoller non autorizza a considerarlo un marxista tout court.

Per quanto riguarda il presunto razzismo e l'atteggiamento antiebraico di Sombart, partiamo da quanto egli rileva nella Prefazione a Die Juden, un'analisi sollecitata dalle riflessioni di M. Weber su 'etica protestante' e 'genesi dello spirito del capitalismo'. Come egli sottolinea: «mi sono imbattuto nella questione ebraica davvero per caso», durante la revisione de Il capitalismo moderno ${ }^{9}$, giungendo al «convincimento» che tra il XV e il XVII secolo «gli Ebrei hanno svolto nella costruzione dell'economia moderna un ruolo infinitamente maggiore di quello che si è soliti riconoscere loro». Ovviamente Sombart, come Weber, è consapevole del fatto che «il capitalismo moderno deve la propria nascita all'associarsi puramente accidentale di popoli dotati di indoli radicalmente diverse, i cui destini sono stati determinati da mille circostanze pure accidentali $\gg^{10}$. Si le», V, 8 (1), 1899, p. 35.

Schmoller, Lineamenti, cit., pp. 147-148.

6 Cfr. ivi, p. 86.

7 W. Sombart, Zur Kritik des ökonomischen Systems von Karl Marx, «Archiv für Soziale Gesetzgebung und Statistik», Siebenter Band, 1894, p. 591.

8 Cfr. ivi, p. 592. Come vedremo, è un giudizio che Sombart riconferma in tutta la sua opera.

9 W. Sombart, Die Juden und das Wirtschaftsleben, Duncker und Humblot, München und Leipzig 1918, (I ed. 1911), p. 19.

10 Cfr. ivi, p. 22 e p. 87. Si veda anche: I.L. Horowitz, The Jews and Modern Communism: the Sombart Thesis Reconsidered, «Oxford Journal», 6 (1), Feb. 1986, p. 14. 
tenga presente, egli continua, che con Die Juden ho costruito un'opera «unilaterale», che «vuole mostrare il ruolo degli Ebrei nella vita economica», utilizzando «tutto e soltanto quel materiale che si riferisce a quest'unico tema, senza menzionare alcuni dei fattori che - al di fuori degli Ebrei e parallelamente ad essi - hanno contribuito alla costruzione del capitalismo moderno». In tal senso, l'opera «non mira a dimostrare la fondatezza di questa o quella concezione della storia, né tende a far prevalere la concezione "razziale" nella vita economica»: la «questione delle razze, e specialmente [...] la "questione ebraica"», non può costituire in maniera assoluta un canone interpretativo «in nessun campo $\gg^{11}$. Non sono accettabili le «dispute verbali» dei «nostri teorici della razza» e sono «campate in aria» tutte le loro osservazioni circa «una specificità, un'identità antropologica» degli ebrei ${ }^{12}$. Tutto ciò, viene ribadito persino in Deutscher Sozialismus ${ }^{13}$.

Il terzo aspetto è relativo ai rapporti tra Sombart e il nazismo. Innegabilmente, nel secondo decennio del XX secolo la riflessione sombartiana assume tratti antiliberali e antidemocratici, manifestando una crescente ostilità nei riguardi dell'economia di mercato. La sua visione dello stato e del rapporto tra stato e società cambia radicalmente, in base alla convinzione che l'Hochkapitalismus segni il definitivo fallimento del progetto liberale e dell'utopia illuministica. Gli automatismi del mercato, determinati dalla sfrenata ricerca del profitto, possono essere piegati verso esigenze sociali solo da orientamenti politici che indirizzino il «gigante cieco» ${ }^{14}$. Questo è ribadito nell'incontro pisano del 1933, quando Sombart sottolinea la necessità di ripristinare, dinnanzi all'aggravamento delle crisi economiche e della 'soziale Frage', forme di controllo politico dell'economia, tese a garantire un più armonioso sviluppo sociale: «Noi dobbiamo capire che l'economia non è il nostro fato [...] L'epoca dell'economia che noi abbiamo vissuta dal secolo XVIII sino alla guerra, sta per risolversi visibilmente in un' "epoca politica"». Essa «farà retrocedere l'economia dalla sua posizione dominante $\gg{ }^{15}$. Questa visione ha, come vedremo, la sua origine nelle analisi sul capitalismo contemporaneo che Sombart sviluppa a partire dal 1910, per cui è arduo attribuirla a convenienze politiche o a calcoli opportunistici, maturati dopo la nascita del nazismo. D’altra parte, come aveva rilevato Schmoller, il

11 Sombart, Die Juden, cit., pp. 24-26.

12 Cfrivi, p. 349.

13 W. Sombart, Deutscher Sozialismus, Buchholz \& Weisswange, Charlottenburg 1934, pp. 189 sgg.

14 W. Sombart, Il Borghese. Lo sviluppo e le fonti dello spirito capitalistico, Longanesi, Milano 1983; ed. or.: Der Bourgeois: zur Geistesgeschichte des modernen Wirtschaftsmenschen, Duncker und Humblot, Leipzig; trad. inglese: M. Epstein, The Quintessence of Capitalism: A Study of the History and Psychology of the Modern Business Man, E.P. Dutton and Company, New York 1915, p. 286.

15 W. Sombart, Intervento nel Convegno di scienze morali e storiche. 14-20 novembre 1932. Tema: l'Europa, vol. I. Atti preliminari - Processi verbali, Reale accademia d'Italia, Roma 1933, p. 403. 
giovane Sombart aveva messo in pericolo la sua carriera accademica per difendere le proprie convinzioni scientifiche, considerate troppo prossime a Marx ${ }^{16}$, dimostrando che egli si lasciava guidare (al di là dei loro limiti) esclusivamente dalle sue idee scientifiche. Certo, dati gli esiti della sua riflessione, riteneva di poter avere riconoscimenti più ampi dal regime nazista, ma va ricordato che $i$ suoi rapporti con i gerarchi nazisti (1932-1933) furono intesi in primo luogo a salvaguardare l'autonomia scientifica del Verein (di cui era diventato presidente). Non avendo ottenuto garanzie in tal senso, preferì lo scioglimento del Verein ${ }^{17}$. Insomma, se non si può considerare «il Sombart della prima maniera "teorico del marxismo" [...] così è impossibile definirlo, a scopo di omaggio o no, di elogio o di biasimo secondo le tendenze e gli orientamenti politici "teorizzatore del nazional-socialismo" ${ }^{18}$.

3. II movimento socialista e Der moderne Kapitalismus (1902): i fattori soggettivi e il presupposto materialistico del loro sviluppo

Le dieci edizioni (anche se con diversi titoli) di Sozialismus und soziale Bewegung im 19. Jahrhundert definiscono con notevole precisione la parabola della riflessione scientifica e della visione politica di Sombart. Sino al 1910 Sombart si muove in un orizzonte analitico piuttosto omogeneo. Il 'moderno capitalismo' viene esaminato entro due prospettive complementari: sul piano politico gli studi sulla 'soziale Bewegung' riflettono sulle trasformazioni del capitalismo contemporaneo e sulla possibile transizione al socialismo; sul piano più propriamente scientifico, in Der moderne Kapitalismus (1902), Sombart analizza i tratti distintivi del moderno capitalismo, a partire dalla transizione dal Medioevo al nuovo sistema economico. Dopo il 1910, sino a Deutscher Sozialismus (1934), gli studi sul 'movimento sociale' si muovono in un orizzonte teorico diverso, segnato dal disincanto e dalla convinzione che i caratteri del sistema economico (data la forza dei suoi meccanismi endogeni e gli effetti negativi e irreversibili che il capitalismo ha prodotto sull'uomo e sulla società) non potranno mutare neanche nel passaggio dal capitalismo al socialismo. Queste convinzioni emergono anche nell'analisi sviluppata nella seconda edizione di Der moderne Kapitalismus.

Nelle prime edizioni degli studi sul movimento operario, Sombart insiste, marxianamente, sul rapporto tra sviluppo delle forze produttive, crescita della

16 A. Mitzman, Sociology and Estrangement. Three Sociologists of Imperial Germany, Alfred A. Knopf, New York 1973, pp. 158 sgg. Com'è noto, per la sua prossimità a Marx e a Lassalle e per la sua eterodossia, 'der rote Professor' non ottenne la cattedra in Germania e fu confinato a Breslavia. Solo nel 1917 ebbe a Berlino la cattedra, appartenuta a Adolph Wagner, che tenne fino al momento del suo pensionamento (1931). Gli subentrò J.A. Schumpeter.

17 R. Rie $\beta$, Werner Sombart under National-Socialism. A First Approximation, in J. Backhaus (ed.), Werner Sombart (1863-1941). Social Scientist, Metropolis-Verlag, Marburg 1966, vol. I, p. 196.

18 A. Sapori, Werner Sombart (1863-1941). Lettura tenuta il 3 maggio 1943, Le Monnier, Firenze 1944, p. 12. 
soggettività proletaria e graduale modificarsi dei 'rapporti di potere' tra borghesia e proletariato, sostenendo la necessità di un approccio epistemologico innovativo (e materialisticamente fondato) nell'analisi del 'moderno capitalismo'. Sombart esplicita questo approccio in una accesa riunione del Verein a Breslau (che egli stesso aveva organizzato nel 1899), contrapponendosi alla visione dei fondatori del Verein e alla cosiddetta 'scuola etica' ${ }^{19}$. In quell'occasione, Sombart espresse la convinzione che le condizioni materiali di esistenza dovessero essere considerate come un prius logico, rispetto ai sistemi di valori e alla cultura, poiché anche la dimensione etica e culturale è connessa con lo sviluppo delle forze produttive: «voler essere morale al costo del progresso economico rappresenta l'inizio della fine dell'intero sviluppo della cultura $\gg^{20}$. Come aveva affermato nel 1897: «una sana politica sociale si deve impegnare a dare il massimo sostegno alle classi sociali che rappresentano il progresso economico, poiché con ciò il loro ideale, il più alto sviluppo delle forze produttive, può essere realizzato»e questo è da considerarsi necessario proprio «nell'interesse del progresso della cultura $\gg^{21}$. Questo approccio, condiviso dalla generazione più giovane presente nel Verein für Sozialpolitik (i fratelli Weber, Jaffé, Lederer ecc.) ${ }^{22}$, insisteva perciò sulla esigenza di analizzare la relazione tra condizioni «oggettive» del sistema economico e dimensione «psicologica» e culturale degli attori sociali nei processi di trasformazione della società ${ }^{23}$.

Tuttavia, Sombart non sottovaluta i limiti analitici del materialismo storico e le distorsioni che il suo 'radicale oggettivismo' avevano prodotto nella lettura del cambiamento sociale. Il banale determinismo economicistico, che la profonda cultura filosofica ed economica di Marx - al di là delle sue premesse metodologiche - aveva saputo scongiurare, era ormai diventato il tratto distintivo del marxismo. Non casualmente, la teoria del crollo economico (die Zusammenbruchstheorie) e l'interpretazione delle crisi economiche come memento mori del capitale (à la Kautsky), erano considerati il reale presupposto della nascita del socialismo ${ }^{24}$. Ma, commenta Sombart, nella storia «non esistono necessità asso-

19 Sombart non prende solo le distanze da Schmoller e da molti dei fondatori del Verein, ma anche dall'approccio di suo padre, stimato componente del Verein.

20 W. Sombart, Dennoch. Aus Theorie und Geschichte der gewerkschaftlichen Arbeiter-Bewegung, Gustav Fischer, Jena 1900, pp. 85 sgg.

21 W. Sombart, Ideale der Sozialpolitk, «Archiv für soziale Gesetzgebung und Statistik», Bd. 10, 1897, p. 44. Cfr. anche F. Lenger, Werner Sombart: 1863-1941. Eine Biographie, Verlag C.H. Beck, München 2012, pp. 98 sgg.

22 F. Lenger, Sozialismus und soziale Bewegung (auch in Italien): Sombart Marx und Michels 1892 bis 1908, in Id. Sozialwissenschatf um 1900, Peter Lang, Francoforte 2009; cfr. anche Lenger, Werner Sombart: 1863-1941, cit., pp. 71-78.

${ }^{23}$ Questo approccio e i temi che esso implicava portò alla pubblicazione dell'Archiv für Sozialwissenschaft und Sozialpolitik. Si vedano, a tal proposito, le interessanti riflessioni del Geleitwort del 1904 dell'Archiv für Sozialwissenschaft und Sozialpolitik, vol. 21, pp. I-VII. Sul ruolo dell'Archiv cfr. S. Whimster, Max Weber: Work and Interpretation, in G. Ritzer, B. Smart, Handbook of Social Theory, Sage Publications, London 2001, spec. pp. 55 sgg.

24 W. Sombart, Socialism and Social Movement, trad. Epstein, J.M. Dent, New York 1909, p. 86. 
lute», dedotte esclusivamente dalla logica interna del sistema economico, senza considerare il contributo decisivo dei fattori soggettivi. Assurdo «applicare alla vita sociale l'idea dei processi dei fenomeni naturali che si svolgono automaticamente», dimenticando che «tutti i fenomeni sociali sono determinati e realizzati da uomini», che agiscono «ponendosi finalità e cercando di perseguirle» in ambienti sociali definiti da specifiche configurazioni culturali e peculiari assetti politico-istituzionali. Se il socialismo è «necessario, ineludibile», questo dipende dalla capacità del movimento operaio di indirizzare lo sviluppo, determinato dalla crescita delle forze produttive, verso un'organizzazione dell'economia fondata su principi di equità sociale. Infatti il movimento operaio sostituisce al movente meramente «materiale», che ha caratterizzato la borghesia, un movente «ideale», incentrato sulla creazione di istituti democratici atti a garantire, nelle situazioni date, concreti processi di emancipazione umana ${ }^{25}$.

In tal senso, Sombart manifesta la sua ammirazione per il 'modello inglese', «stadio inevitabile del nostro futuro sviluppo», perché al suo interno le tradeunions hanno saputo ricondurre i contrasti tra le classi nell'alveo delle dinamiche delle istituzioni democratiche ${ }^{26}$. Tale vocazione riformista ha garantito lo sviluppo delle forze produttive e ha modificato in profondità le relazioni industriali, introducendo un vero «costituzionalismo industriale contro l'assolutismo o il feudalesimo industriale» della fase precedente, contribuendo «involontariamente a portare, attraverso un organico processo di trasformazione, il sistema economico capitalista verso più elevati livelli di organizzazione sociale $\gg^{27}$. Lo «spirito faustiano» e la 'frenesia', che avevano caratterizzato la borghesia in un contesto di «inquieto, continuo, progressivo movimento» finalizzato all' incremento della ricchezza materiale, sono stati ereditati dal proletariato, che, animato da «finalità $[\ldots]$ ideali», contribuirà a costruire una società più equa ${ }^{28}$.

Entro questo orizzonte analitico si inscrive la prima edizione di Der moderne Kapitalismus. Il tema della genesi e dello sviluppo del capitalismo moderno prende l'avvio da una più robusta riflessione epistemologica, tesa a costruire uno «storicismo teoretico ${ }^{29}$, che superasse la contrapposizione tra «teoria ed empiria», analisi teorica e ricerca empirica. Gli interminabili dibattiti che si erano sviluppati su tale contrapposizione, incluso il Methodenstreit, hanno consolidato l'idea della inconciliabilità tra modelli di «spiegazione astratta e generale» e modelli di spiegazioni fondati sulle «indagini empiriche ${ }^{30}$. Solo la costruzione di una scienza sociale teoretica, fondata su «un unitario principio

Cfr. ivi, pp. 317-318.

Cfr. ivi, pp. 147 sgg.

7 Sombart, Dennoch. Aus Theorie und Geschichte, cit., pp. 86-87.

28 Sombart, Socialism and Social Movement, cit., pp. 317-318.

29 F. Lenger, Ethics and Economics in the Work of Werner Sombart, in P. Koslowski (ed.), Methodology of the Social Sciences, Ethics, and Economics in the Newer Historical School, Springer, Berlin-New York 1997, p. 148.

30 W. Sombart, Der moderne Kapitalismus, Duncker und Humblot, Leipzig 1902, p. X. 
esplicativo» (Erklärungsprinzip) nell'organizzazione del materiale empirico ${ }^{31}$ può contribuire al superamento dell'idea che analisi teorica e ricerca empirica siano «in una ostile opposizione» (《in einen feindlichen Gegensatz») $)^{32}$. Si tratta, in sostanza, di adottare il «postulato radicale di una spiegazione uniforme a partire dalle cause ultime», secondo il modello marxiano ${ }^{33}$, mettendo a fuoco le determinanti «oggettive» specifiche del sistema economico e l'insieme delle trasformazioni culturali e 'psicologiche' degli attori sociali che ne hanno determinato genesi e sviluppo.

La messa a fuoco dell'intima relazione tra i cambiamenti tecnici nell'organizzazione produttiva e del commercio e le trasformazioni culturali e dei sistemi di valori, nel contesto della nascente economia di mercato, consentirà di cogliere quella «serie di circostanze» (di ordine materiale e culturale) che determinano il crollo delle vecchie concezioni della ricchezza, nonché degli stili di vita del passato. Scompaiono anche le visioni «magiche» del mondo che avevano sostenute quelle concezioni dell'uomo e della società. Si comprenderanno allora le ragioni per cuil'incarnazione (Menschwerdung) dello «spirito del capitalismo» abbia via via orientato le attività dei «migliori bottegai» (die bessere Krämern), moltiplicando rapidamente il numero delle organizzazioni economiche che si muovevano nel circuito dei piccoli usurai (in den Kreise der Winkelwucherer) e facendo convergere una serie eterogenea di attività verso modalità di arricchimento di tipo capitalistico ${ }^{34}$. Secondo Sombart, non è casuale che il moderno spirito acquisitivo si sia sviluppato in primo luogo negli ambienti in cui la coesione comunitaria e le vecchie tradizioni erano più deboli: i circuiti del commercio con gli stranieri o le attività economiche di gruppi sociali, come quello degli ebrei che, in virtù della loro religione o della loro posizione di oppressi, non erano parte organica delle comunità in cui operavano. Solo successivamente, esso si è diffuso tra le masse popolari, divenendo la forza motrice di comportamenti e attività che hanno infranto «la struttura del vecchio mondo aristocratico», avviando quel processo di democratizzazione, che rappresenta «il peculiare fondamento della società moderna $\gg^{35}$.

Come si vede, Sombart, pur enfatizzando una serie di importanti precondizioni (crociate, nuovi sistemi di comunicazione, miglioramento delle tecnologie ecc.), in grado di sostenere l'ipotesi del superamento dei vecchi sistemi produttivi in direzione del capitalismo, accenna a una diversa prospettiva analitica, non ritenendo probabilmente soddisfacente l'idea «that the capitalist of the later

${ }^{31}$ Cfr. ivi, p. XIII.

32 Cfr. ivi, pp.X-XI.

33 Tuttavia, nella Prefazione alla seconda edizione Sombart deve ammettere che le critiche ricevute, relativamente ad una certa indeterminatezza metodologica, avevano un fondamento, dal momento che si è impegnato ad evitare il 'peggior difetto' della prima edizione: «l'inammissibile miscuglio di considerazioni teoretiche ed empirico-realistiche» (W. Sombart, Il capitalismo moderno, Utet, Torino 1978, p. 85).

34 Sombart, Der moderne Kapitalismus, cit, pp. 388-389.

35 Cfr. ivi, pp. 389 sgg. 
centuries was the direct descendant of the merchant of the guild $\gg^{36}$. Il riferimento a figure 'esterne' o marginali (stranieri, eretici, ebrei) rispetto alla storia delle comunità occidentali, sembra svolgere una funzione peculiare: evidenziare modelli di soggettività che, per loro caratteristiche intrinseche, contribuiscono a dare un tono specifico allo 'spirito economico'. Sombart sembra accennare a una sorta di divaricazione tra una possibile evoluzione 'naturale' delle comunità occidentali, fondata su determinanti endogene del sistema medievale, e un'evoluzione 'innaturale', connessa con l'attivismo di fattori soggettivi e moventi psicologici esterni rispetto alla storia delle comunità medievali. Tuttavia, nella prima edizione di Der moderne Kapitalismus, Sombart ridimensiona la rilevanza di quest' ultima prospettiva analitica, rilevando, a proposito degli ebrei, che la loro influenza non va sopravvalutata, a causa della loro limitata consistenza quantitativa nelle aree in cui il capitalismo ha avuto il suo primo sviluppo. Successivamente, tale prospettiva acquisterà un ruolo centrale nell'analisi di Sombart.

\section{Il capitalismo maturo: la nuova ricerca sul ruolo dei fattori soggettivi} nella dinamica sociale

All'indomani del 1910 Sombart riflette sulle drammatiche trasformazioni del sistema economico dei suoi tempi e sulle loro conseguenze sulla dinamica sociale. Tali riflessioni sono accompagnate dalla percezione della crisi irreversibile della «missione tedesca $»$ e degli ideali umanistici e universalistici che essa aveva rappresentato ${ }^{37}$ : l'utopia illuministica che aveva orientato parte rilevante della cultura europea $<$ was increasingly dismantled in Europe $\gg^{38}$. Opere come Die Juden und das Wirtschaftsleben (concepita come una risposta a Die protestantische Ethik und der Geist des Kapitalismus), Die Zukunft der Juden (1912), Der Bourgeois (1913), Studien zur Entwicklungsgeschichte des modernen Kapitalismus (I: Luxus und Kapitalismus; II: Krieg und Kapitalismus, 1913), Händler und Herden (1915), che precedono la seconda edizione di Der moderne Kapitalimus (19161917, 1927), evidenziano disincanto e un radicale mutamento nelle prospettive analitiche di Sombart. Si allontanano dalla scena la funzione progressiva del capitalismo e quella del movimento operaio. Il Frühkapitalismus si era giovato della funzione 'progressiva' della borghesia. Successivamente, l'economia capitalistica aveva ricevuto un impulso determinante dallo storico concretizzarsi dei moventi 'ideali', che avevano orientato la strategia politica del proletariato. Come sottolinea nel 1913, anche gli imprenditori del Frühkapitalismus erano orientati dalla ricerca del profitto, ma lo facevano nel rispetto di specifiche esi-

36 A.S. Johnson, Review of Der modern Kapitalismus, «Political Science Quarterly», 18 (2), 1903, pp. 354-356.

37 W. Sombart, Die deutsche Volkswirtschaft im Neunzehnte Jahrhundert (V ed.), G. Bondi, Berlin 1921, p. V.

38 F. Reheis, Return to the Grace of God. Werner Sombart's Compromise with National Socialism, in Backhaus (ed.), Werner Sombart, cit., vol. I, pp. 173-179. 
genze comunitarie. Persino il ritmo del progresso tecnico sottostava ai vincoli etici e comunitari ${ }^{39}$. Ora, quei moventi etici della borghesia 'vecchio stile', seppure non predominanti, sono ormai infranti e i moventi ideali del proletariato, che sembravano poter dare un'anima al capitalismo, non sono più in grado di contrastare la vocazione materialistica e tecnico-razionale del sistema economico. Il confronto con Weber lo porta a ritornare sul ruolo degli ebrei con un'ottica differente: quella di ridefinire i tratti distintivi dell'Hochkapitalismus e dello Spätkapitalismus. Ciò che interessa Sombart è dimostrare che l'agire economico degli ebrei ha dato un contributo fondamentale non solo alla dissoluzione delle vecchie istituzioni economiche, ma anche (e soprattutto) alla diffusione dello 'spirito del capitalismo': «trovo nella religione ebraica la stessa idea guida (dieselben leitenden ideen) che caratterizza il capitalismo». Ebraismo e capitalismo sono intrisi dallo stesso spirito (von demselben Geiste erfüllt) ${ }^{40}$. Insomma, «l'ebraismo è capitalista» e il capitalismo avanzato è «per molti aspetti una manifestazione dello spirito ebraico ${ }^{41}$.

Si tratta di un'evidente discontinuità rispetto alle conclusioni cui era pervenuto su questo tema nella prima edizione di Der moderne Kapitalismus. Se è vero che, dato il modello di soggettività che Sombart ha in mente (su cui torneremo più avanti), egli con riferimento alla religione ebraica e alla «situazione sociale degli ebrei» (degli eretici, e degli stranieri) tenta di «risolvere una delle maggiori difficoltà della sua teoria sull'origine del capitalismo: l'identificazione di una situazione sociale che spieghi la formazione in certi individui e gruppi di atteggiamenti devianti rispetto ai valori tradizionali delle rispettive società e di motivazioni favorevoli all'emergere di una nuova mentalità economica ${ }^{42}$. Tuttavia, è altrettanto vero che questo orientamento diventa decisivo solo nel corso della sua riflessione sull'Hochkapitalismus. L'Hochkapitalismus, infatti, mette in crisi quei processi di democratizzazione e di emancipazione umana che Sombart aveva considerato come «peculiare fondamento» delle nuove relazioni economiche. Il senso di questo cambiamento è chiaro nei capitoli 58-62 del primo volume della seconda edizione di Der moderne Kapitalismus, laddove Sombart si sofferma sugli imprenditori borghesi, i fondatori, gli eretici, gli stranieri, gli ebrei. Infatti, mentre l'imprenditore borghese sviluppa il suo talento nelle attività economiche nel contesto di una visione del mondo che tende a conservare $\mathrm{i}$ valori comunitari e i principi etici che ne caratterizzavano lo stile di vita («virtù borghesi»), tutto ciò non accade nell'agire economico degli altri gruppi sociali (eretici, stranieri o ebrei), esclusi dalla piena partecipazione alla vita pubblica.

39 Sombart, Il Borghese, cit., pp. 127-128.

40 Sombart, Die Juden, cit., p. 242.

${ }^{41}$ Cfr. ivi, p. 187. Su questo cfr. V. Gioia, Capitalism and Judaism in Werner Sombart: a Contribution to the Analysis of Capitalist Rationality and its Limits, «Research in the History of Economic Thought and Methodology», 32, 2014, pp. 15-38; F. Rizzo, Werner Sombart, Liguori, Napoli 1974; M.L.P. Franco, La noción de "espiritu" en las sociologías de Werner Sombart y Max Weber, «Sociólogica», 59, 2005, pp. 27-59.

42 A. Cavalli, Introduzione a Sombart, Il capitalismo moderno, cit., p. 22. 
Per essi, il successo economico appare come l'unico orizzonte possibile di autoemancipazione, indipendentemente dagli effetti che esso determinava sulle comunità di riferimento. Per questo, «l'elemento umano dell'eresia», che aveva rappresentato «uno dei luoghi di nascita del ceto imprenditoriale ${ }^{43}$, è diventato l'elemento caratterizzante del capitalismo contemporaneo. Il 'caso' degli ebrei assume un ruolo paradigmatico:

1. l'ebreo si caratterizza come «puro business man», rivendicando nell'agire economico l'esclusivo «predominio del guadagno, del profitto e dell'interesse contro ogni finalità naturale $\gg^{44}$;

2. la sua peculiare posizione sociale, i caratteri della sua religione, l'indifferenza nei riguardi dei valori comunitari ne fanno il portatore dell' $\ll$ autentico spirito del capitalismo ${ }^{45}$;

3. avendo disgregato il fondamento 'naturale' della vecchia struttura economica e sociale, impone, al di là delle proteste degli uomini d'affari cristiani (der christlichen Geschäftsleute) ${ }^{46}$, l'idea del carattere unidimensionale dell'uomo d'affari: negli affari si è solo uomini d'affari (in Geschäften Nur-Geschäftsmann) ${ }^{47}$;

4. non esistendo «più vincoli, né considerazioni, siano morali, estetiche o sentimentali», l'indifferenza per la spaventosa dilapidazione di energie umane imposte dal «ritmo folle» della dinamica economica diviene il tratto distintivo del capitalismo avanzato ${ }^{48}$.

Siccome il sistema economico è ormai un 'puro meccanismo', che si sviluppa esclusivamente in base a fattori endogeni, indifferente rispetto a ogni movente etico e ideale, non «possiamo più credere alla forza creatrice del capitalismo, come poté fare Marx per il fatto di essere all'inizio del cammino ${ }^{49}$.

Marx - egli continua - disse la prima orgogliosa parola sul capitalismo, in quest'opera ci proponiamo di dire l'ultima modesta parola su questo sistema economico, nella misura in cui viene considerato esclusivamente dal punto di vista economico. Allora era l'alba e cantava l'allodola, oggi ci avviamo verso la sera e le nottole di Minerva incominciano il loro volo. Se vogliamo indicare il rapporto di quest'opera con quella di Marx in una parola e fuori di

43 Sombart, Il capitalismo moderno, cit., pp. $272 \mathrm{sgg}$.

44 Sombart, Die Juden, cit., p. 155.

45 Sombart, Die Juden, cit.; si veda in particolare il cap. VII, pp. 136-180. F. Lenger, Marx, the Crafts, and the First Edition of Modern Capitalism, in Backhaus (ed.), Werner Sombart, cit., vol. II, pp. 35 sgg.; F. Lenger, Ethics and Economics, cit., pp. 147-163; D. Schneider, Sombart or Spirit of Accountability of Capitalism as "Entusiastic Lyricism", in Backhaus (ed.), Werner Sombart, cit., vol. II, pp. 35 sgg.

46 Sombart, Die Juden, cit., p. 151.

47 Cfr. ivi, p. 155.

48 Sombart, Il Borghese, cit., p. 130 e pp. 142-145.

49 Sombart, Il capitalismo moderno, cit., p. 491. 
metafora, potremmo forse dire che essa rappresenta il disincantamento di Marx. Disincantamento significa tuttavia, nel senso positivo che attribuisco al termine, scientificizzazione $\mathrm{e}^{50}$.

Ne deriva che:

1. $\mathrm{i} \ll$ fattori soggettivi», che avevano determinato la genesi e lo sviluppo di questo 'meccanismo', ormai non sono in grado di modificarne la logica ${ }^{51}$;

2. il rapporto tra proletariato e borghesia, prima considerato come fattore di progressiva trasformazione in senso democratico della vita pubblica e dell'organizzazione del lavoro, ora non pare più in grado di garantire i medesimi risultati. Lo stesso proletariato ha ormai smarrito le sue aspirazioni all'emancipazione umana e alla costruzione di una società più equa, limitandosi a perseguire solo finalità materiali: l'incremento dei salari, l'allargamento dei consumi e il miglioramento del livello di benessere materiale ${ }^{52}$;

3. l'ideale del socialismo ha perso la sua funzione progressiva, poiché, esso come economia pianificata - non sarà che il risultato dell'ulteriore perfezionamento dei processi di razionalizzazione tipici del capitalismo: «dobbiamo abituarci al pensiero che la differenza tra un capitalismo tecnicizzato e razionalizzato non è molto grande e che perciò per il destino degli uomini e della loro cultura, è piuttosto indifferente se l'economia del futuro sarà capitalista o socialista ${ }^{53}$. Insomma, rileva amaramente Sombart: «allora, come oggi e nel prossimo e probabilmente anche più vicino futuro, dominava e domina il tardo capitalismo e il proto-socialismo, che sono la medesima cosa» (der Spätkapitalismus und der Frühsozialismus, die dasselbe sind) $)^{54}$.

Le conclusioni di Sombart sono devastanti proprio con riferimento alla sua analisi sul ruolo determinate dei fattori soggettivi (seppure materialisticamente e storicamente connotati) nello sviluppo dell'economia e della società:

Quel che occorreva al capitalismo per raggiungere i suoi scopi era un nuovo genere umano, uomini che fossero in grado di inserirsi in un grande complesso, un'impresa capitalistica o addirittura una fabbrica, in uno di quei meravigliosi sistemi di superiorità, inferiorità e adiacenza, queste strutture artefatte composte da frammenti di uomini. La nuova struttura economica richiedeva questi segmenti umani: esseri senz’anima, spersonalizzati, capaci di essere membri o meglio piccole ruote di un intricato meccanismo ${ }^{55}$.

50 Cfr. ivi, p. 492.

51 T. Parsons, 'Capitalism' in Recent German Literature: Sombart and Weber, «Journal of Political Economy», 36 (6), 1928, p. 651.

52 W. Sombart, Der moderne Kapitalismus, vol. III. Das Wirtschaftsleben im Zeitalter des Hochkapitalismus, Duncker und Humblot, München-Leipzig 1927, vol. I, pp. 424 sgg. Si veda anche Rizzo, Werner Sombart, cit., pp. 43 sgg.

53 Sombart, Il capitalismo moderno, cit., p. 589.

54 Sombart, Die Deutsche Volkswirtschaft im Neunzehnte Jahrundert, cit., p. VI.

s5 Sombart, Il capitalismo moderno, cit., p. 687. 


\section{Gli italiani e Sombart}

L'Italia ha accolto sempre con interesse le analisi del sociologo tedesco. Lo dichiarava lo stesso Sombart che, nel 1933, al margine dei festeggiamenti tributatigli per il cinquantenario della sua iscrizione all'Università di Pisa, rilevava: «in Italia ho trovato maggiore comprensione che altrove, e per primo dal Luzzatto, che dopo aver tradotto il mio Capitalismo lo ha definito senza restrizioni un'opera di storia». Inoltre, egli aggiungeva, i rilievi critici rivolti alla mia opera «non mi hanno lasciato l'amaro in bocca», in quanto fondati sui «documenti dei $[\ldots]$ ricchissimi archivi» sparsi per l'Italia ${ }^{56}$. In sostanza, Sombart ammette che la sua opera in Italia è stata generalmente giudicata senza pregiudizi. Tra gli autori che si sono soffermati, spesso con ampie analisi, sull'opera sombartiana: Achille Loria, Arturo Labriola, Cognetti de Martiis, Gioacchino Volpe, Gino Luzzatto (che nel 1925 traduce Der moderne Kapitalismus), Antonio Labriola, Benedetto Croce, Alberto Bertolino, Armando Sapori, Delio Cantimori, Amintore Fanfani, Gustavo Del Vecchio e altri. Anche di recente il pensiero di Sombart è stato ripreso e rivisitato da diversi studiosi ${ }^{57}$, che hanno enfatizzato il fatto che Sombart (al di là degli esiti contraddittori della sua opera) rimane un importante classico del pensiero economico e sociologico. Rispetto alla rivisitazione attuale può persino apparire sorprendente l'interesse per Sombart nei primi decenni del XX secolo, specie se si considera l'ostilità della cultura economica, sociologica e filosofica, maturata sin dall'ultimo quarto dell'Ottocento, nei riguardi dei rappresentanti della GHS ${ }^{58}$.

56 Sapori, Werner Sombart, cit., pp. 16-17.

57 Sombart in Italia continua ad essere studiato con un certo interesse. Si pensi al convegno Sombart e l'Italia, tenuto a Roma nel 2008, a cura del Deutsches Historiches Institut Rom e della Fondazione Basso, oppure al convegno, organizzato dall'Università del Salento, Il Borghese di Sombart: cento anni dopo. Dalle riflessioni condotte in questo convegno sono state originate due pubblicazioni: lo special issue di «Dada. Rivista di antropologia post-globale», del 2015 dal titolo Sombart's Thought Revisited e la sezione monografica sul Borghese di Sombart della rivista «Sociologia italiana. AIS Journal of Sociology», n. 5 , aprile 2015. A ciò si aggiunge la recente pubblicazione, da parte di giovani studiosi, di diversi saggi del sociologo tedesco: W. Sombart, Le origini della sociologia, a cura di Silvia Fornari, Armando, Roma 2009; Id., Tecnica e cultura, a cura di Gennaro Iorio, Kurumuni, Martignano 2012; Id., Mercanti ed eroi, prefazione di Francesco Ingravalle, Aracne, Roma 2012, e nel 2014 un'altra edizione dello stesso saggio, a cura di Fabio Degli Esposti, Ets, Pisa. Inoltre, Roberta Iannone ha curato la pubblicazione di Saggio sull'Uomo, con il titolo di Umano, ancora umano. Per un'analisi dell'opera Sull'Uomo di Werner Sombart, Bonanno Editore, Acireale-Roma. Ovviamente, non considero quella letteratura della destra che ha tentato di accreditare l'idea di un Sombart razzista e filonazista anche se resta il rammarico, come osservava Federico Trocini, che alcune delle maggiori opere di Sombart siano «state pubblicate presso case editrici che possono essere fatte rientrare nel composito universo [della] destra radicale» (F. Trocini, La «controversa» fortuna di Sombart in Italia. Un secolo di recensioni, traduzioni e commenti, «Rivista storica italiana», 122 (111), 2010, p. 1045. Si veda in particolare la nota n. 3).

58 Trocini, La «controversa» fortuna di Sombart in Italia, cit., pp. 1045-1087. S. Pisanelli, Werner Sombart and his Reception in Italy, «DADA. Rivista di antropologia post-globale», 
Basti pensare alle aspre polemiche di Francesco Ferrara contro il cosiddetto «germanismo economico» e alle critiche radicali di Vilfredo Pareto ${ }^{59}$. Alle ostilità degli economisti si aggiungevano quelle degli storicisti italiani: Antonio Labriola e Benedetto Croce. Il primo, nei suoi In memoria del manifesto dei comunisti e Discorrendo di socialismo e filosofia, manifestava la sua intolleranza nei riguardi dello storicismo acritico dei rappresentanti della GHS, considerati come «geni di mediocrità», a cominciare da Roscher: «quel famoso emarginatore di note erudite e minute, via via apposte a paragrafi pieni zeppi di definizioni nominali e spesso insensate ${ }^{60}$. Egli sottolinea, inoltre, l'irrilevanza conoscitiva delle loro analisi empiriche ${ }^{61}$, tese a giustificare, con riferimento alla Prussia, «l'illusione di un monarcato sociale, che passando sopra all'epoca liberale, armonicamente risolvesse la cosiddetta questione sociale»: questa «fisima», riproposta «in infinite varietà di socialismo cattedratico, e di stato» aggiunge «alle varie forme di utopismo utopico e religioso [...] una nuova: l'utopia burocratica e fiscale; ossia l'utopia dei cretini ${ }^{62}$.

Altrettanto radicali sono le obiezioni di B. Croce, il quale - attraverso «La critica» - rimproverò ripetutamente ai rappresentanti della GHS la rinuncia all'approccio deduttivo. Tale rinuncia aveva impoverito «la ragione e il ruolo della scienza storica» sul piano speculativo ${ }^{63}$, favorendo il ricorso a pratiche scientifiche tipiche della sociologia (la «inferma scienza») e caratterizzate da inaccettabili forme di empirismo acritico ${ }^{64}$. Come Labriola, Croce enfatizzò anche la mancanza di autonomia rispetto al potere politico prussiano da parte dei rappresentanti della $\mathrm{GHS}^{65}$.

Queste valutazioni critiche non influenzarono, generalmente, la riflessione italiana degli storici economici sull'opera di Sombart e, soprattutto, su Der moderne Kapitalismus. Essa si sviluppò su tre livelli:

special issue, 1, 1915, pp. 155-182. Si veda anche: M.R. Caroselli, Giudizi italiani sull'opera di Werner Sombart, in G. Barbieri et al., L'opera di Werner Sombart nel centenario della nascita, Giuffrè, Milano 1964, pp. 29-62.

59 V. Gioia, Gli economisti italiani e la scuola storica tedesca dell'economia: storia di un equivoco, in P. Barucci (a cura di), Le frontiere dell'economia politica. Gli economisti stranieri in Italia: dai mercantilisti a Keynes, Polistampa, Firenze 2003, pp. 273-306; V. Gioia, Werner Sombart: il capitalismo moderno e il suo futuro. Un'ipotesi di lavoro, «Sociologia italiana. AIS Journal of Sociology», 5, aprile 2015, pp. 11-32.

60 A. Labriola, Discorrendo di socialismo e filosofia, in Id. Saggi sul materialismo storico, a cura di V. Gerratana, A. Guerra, Editori Riuniti, Roma 1968, pp. 189-190.

61 A. Labriola, Del materialismo storico. Dilucidazione preliminare, in Id., Saggi sul materialismo storico, cit., pp. 57-58. Si veda D. Triggiano, Introduzione a Max Weber. Da Economia e società $a$ Sociologia della religione, Meltemi, Roma 2008, p. 190.

62 Labriola, Del materialismo storico, cit., p. 58.

63 B. Croce, La storia come pensiero e come azione, Laterza, Bari 1965, p. 72.

64 R. Michels, The status of sociology in Italy, «Social Forces», 9 (1), 1930, pp. 37-39; A. Scaglia, La sociologia tedesca in Italia, «Studi di sociologia», 29 (2), 1991, p. 161 e Pisanelli, Werner Sombart and his reception, cit., pp. $155 \mathrm{sgg}$.

65 Croce, La storia come pensiero e come azione, cit., p. 69. 
1. sul giudizio di Sombart sul capitalismo italiano;

2. sulle sue scelte metodologiche;

3. sugli esiti della sua analisi circa il 'futuro' del capitalismo.

Sui primi due aspetti c'è una sostanziale convergenza degli studiosi italiani. Sombart è considerato come «uno storiografo di razza», che con «stile» originale «fa risaltare le vecchie e le nuove idee in una luce mai vista e inserisce $i$ fatti prescelti come significativi in una trama dagli insospettati rilievi ${ }^{66}$.Viene apprezzata l'enfasi sombartiana sulla precocità del capitalismo italiano: l'Italia è «la terra dove si è sviluppato per primo lo spirito capitalistico». In Italia, grazie all'adozione di innovativi processi di conduzione razionale delle imprese agricole e all'affermarsi di moderne forme di contabilità, dal XII secolo si sono radicati originali orientamenti culturali e comportamentali, tipici del capitalismo ${ }^{67}$. Nel corso del XIV secolo, lo 'spirito borghese' appare ormai come un fenomeno di massa ${ }^{68}$, imponendosi nelle grandi città marinare, Genova e Venezia, e acquisendo un «più forte impulso» a Firenze e in Toscana, in virtù dei crescenti processi di urbanizzazione che coinvolsero l'intera regione ${ }^{69}$. Tuttavia, aggiunge Sombart, «tanta magnificenza capitalistica giunge [...] abbastanza velocemente alla fine» e «lo spirito dell'impresa decade», anche se permangono «inclinazione al calcolo e "abilità amministrativa"», che continuano a perfezionarsi «durante il sedicesimo e diciassettesimo secolo». L'affievolirsi della forza trasformatrice dello 'spirito del capitalismo' fu determinato dalla diffusione nelle città italiane della «mania nobiliare o, come fu chiamata, della mania d' ispagnolire la vita» ${ }^{70}$.

Questa ricostruzione di Sombart, relativamente ai caratteri dello sviluppo del capitalismo italiano e al suo rapido tramonto, sono contestate dagli studiosi italiani, che la considerano come predeterminata dalla sua inadeguata rappresentazione della vita economica dell'Italia medievale, caratterizzata dalla «piccolezza del commercio» e dal prevalere di una pura logica di «sussistenza» ${ }^{71}$. Sombart realizza, per un'errata scelta metodologica e per un insufficiente uso di

G. Barbieri, Werner Sombart ed il valore dello "spirito economico" nella ricostruzione storiografica, in Barbieri et al., L'opera di Werner Sombart, cit., p. 154. A. Bertolino, Considerazioni critiche sulla metodologia sombartiana, in Barbieri et al., L'opera di Werner Sombart, cit., p. 174. Sapori, Werner Sombart, cit., p. 15.

67 Sombart, Il capitalismo moderno, cit., pp. $633 \mathrm{sgg}$.

68 Si tratta di quel concetto della 'distruzione creatrice', reso famoso da Schumpeter, che Sombart deriva da Marx e che anima profondamente la sua intera opera, caratterizzando quello che egli definisce come 'lo spirito borghese', che «dischiude all'azione dell'uomo, un campo meraviglioso e particolarmente fertile dove l'aspirazione all'infinito, lo spirito d'intrapresa si impongono anche proprio sul terreno dell'attività quotidiana volta al soddisfacimento dei bisogni», cfr. Sombart, Il capitalismo moderno, cit., p. 175.

69 Cfr. ivi, p. 635.

70 Sombart, Il borghese, cit., p. 103.

71 Sapori, Werner Sombart, cit., p. 21; A. Sapori, La cultura del mercante medievale italiano, in G. Airaldi (a cura di), Gli orizzonti aperti. Profili dei mercanti medievali, Paravia, Torino 1997, pp. 139-173; Barbieri, Werner Sombart e il valore dello "spirito economico", cit., pp. 154 sgg. 
materiale documentale e archivistico, un «lungo, minuzioso e [...] tenace lavoro di minimizzazione degli scambi medievali $>^{72}$. Inoltre, proietta i tratti di questa sua rappresentazione sui secoli immediatamente successivi ${ }^{73}$, non avvedendosi degli elementi di continuità tra l'evoluzione dei sistemi economici medioevali e quelli dei secoli successivi. Non percepisce, ad esempio il fatto che, già nel corso del Quattrocento, il mercante «non si muove più nell'ambito angusto dell'economia cittadina, ma ha nelle sue mani tutto il traffico internazionale, che si esercitava in quei tempi nei paesi d'Occidente $>^{74}$.

Il fatto è che la visione sombartiana, costruita in analogia con «quanto avveniva nelle zone tedesche ancora dominate dalla feudalità», trascurava l'ampiezza di traffici, le innovazioni istituzionali, la ricchezza culturale che emergevano in città come Firenze, Pavia, Cremona e Milano o in aree come la Toscana e la Lombardia, che già in epoca medioevale erano stabilmente entrate a far parte del «concerto crescente dei centri economici più avanzati» ${ }^{75}$. Inoltre, Sombart ignora completamente la rilevanza, continuità e ricchezza dei traffici delle città costiere dell'area del Mediterrane ${ }^{76}$. Sono completamente trascurati i caratteri innovativi della «rapida ascesa» di Milano, «nella seconda metà del Trecento», dovuta al ruolo svolto dai «magni mercatores» e dai «lanaioli »: figure che andavano - a tutti gli effetti - considerate tipologie di moderne «figure imprenditoriali». I primi erano riconosciuti e stimati «controllori del traffico internazionale $[\ldots]$ potenti sino al punto di mantenere e sviluppare le vie di comunicazione»; i secondi furono «creatori delle manifatture tessili [...] con più di cinquecento organismi produttivi nella seconda metà del secolo XIV». Insomma, un vero nucleo vitale di quella «solida borghesia [che] sostiene finanziariamente la dominazione viscontea $\gg^{77}$.

Questa sottovalutazione si accompagna ad un'altra, altrettanto significativa, relativa al radicamento di una cultura di intrapresa, che sosteneva crescita e consolidamento delle nuove forme di «agire economico» ${ }^{78}$. Esso è documentato da una vasta letteratura 'minore', che Sombart non considera o considera in modo episodico ${ }^{79}$, limitandosi a riproporre l'esempio classico di Leon Battista Alberti, le cui opere finiscono con l'essere valutate come un'eccezione o un monumento

72 F. Melis, Werner Sombart e i problemi della navigazione nel Medioevo, in Barbieri et al., L'opera di Werner Sombart, cit., p. 87.

73 Ibidem.

74 G. Luzzatto, Dai servi della gleba agli albori del capitalismo. Saggi di storia economica, Laterza, Bari 1966, p. 519 e A. Sapori, Studi di storia economica. Secoli XIII, XIV, XV, vol. I, Sansoni, Firenze 1955, pp. 495-533.

75 Barbieri, Werner Sombart ed il valore dello "spirito economico", cit., p. 155 e p. 159.

76 Sapori, Werner Sombart, cit., pp. 24-25.

77 Ivi, p. 160.

78 Sombart, Il capitalismo moderno, cit., pp. 137 sgg. e pp. 314-316. Si veda anche Sapori, La cultura del mercante medievale, cit., pp. $145 \mathrm{sgg}$.

79 Sapori, Werner Sombart, cit., p. 27; Barbieri, Werner Sombart ed il valore dello "spirito economico", cit., p. 165. 
isolato in un ambiente culturalmente povero e stagnante. Tuttavia, non hanno fondamento né l'idea «che il mercante medievale fu non solo incolto, ma generalmente illetterato», né quella secondo cui il Medioevo italiano fu caratterizzato da «una folla di piccoli uomini d'affari, i quali si dedicarono, con mentalità puramente artigiana, ad una limitata attività, bastevole a dar loro i mezzi per una vita modestissima $\gg^{80}$. Al contrario, la documentazione archivistica consente di ricostruire, dal Duecento in poi, le attitudini del «mercante italiano» ad annotare, ordinare e conservare metodicamente tutto ciò che era ritenuto interessante per le sue attività, evidenziando un'elevata cultura generale e una «cultura matematica adeguata» alle esigenze dei nuovi sistemi di produzione e commercio $^{81}$. Tale atteggiamento era favorito dalla specifica «costituzione dei Comuni italiani», attraverso cui si diffonde in frazioni cospicue della popolazione, chiamata a prendere «parte attiva alla vita pubblica» e a dimostrare «in ogni ufficio, di possedere qualità non trascurabili $>^{82}$.

In realtà, tutto questo è stato possibile perché Sombart, adottando come modello il peculiare sviluppo capitalistico dell'Europa del Nord, non sembra interessato a ricostruire la specificità del capitalismo italiano, bensì ad «inquadrare ad ogni costo anche il caso italiano nello schema di interpretazione e sviluppo di tutto il precapitalismo europeo». Egli solo episodicamente enfatizza qualche tratto distintivo del nascente capitalismo italiano, attribuendogli però un carattere di eccezionalità che non smentisce la regola ${ }^{83}$. Sombart stesso sembra confermare questo giudizio, facendo riferimento al suo tentativo (e alla sua impazienza) di rappresentare unitariamente «il tipo storico» del capitalismo europeo, trascurando le differenze che si manifestavano al suo interno, quando rileva: «la vita economica del futuro» ci porrà in presenza di modelli di capitalismo che, costruiti da popolazioni extra-europee, si affrancheranno parzialmente dal modello europeo-americano e costruiranno un «loro capitalismo». Dunque,

il capitalismo dei cinesi, dei malesi, dei negri non sarà più un 'capitalismo moderno' quale quello da me trattato in quest'opera. Sarà un sistema che porterà alcuni tratti del capitalismo europeo-americano, ma che sarà nella sua essenza radicalmente diverso dal nostro, poiché diversi saranno i principi sui quali si fonderà. Bisogna tuttavia sempre tener presente che anche manifestazioni di civiltà, come il capitalismo moderno, non meno che le culture dei popoli, sono individui storici che compaiono nella storia una volta sola. Questa del resto è stata l'idea fondamentale che mi ha guidato nella stesura di quest'opera ${ }^{84}$.

Questo approccio, adottato per il futuro del capitalismo, sarebbe dovuto essere, a maggior ragione, adottato in riferimento al passato, quando i tratti differen-

1 Cfr. ivi, p. 148 e pp. 647 sgg.

82 Cfr. ivi, p. 150.

83 Barbieri, Werner Sombart ed il valore dello "spirito economico", cit., p. 155.

${ }^{84}$ Sombart, Il capitalismo moderno, cit., p. 857. 
ziali tra le diverse aree erano ancora più marcati. D'altronde, non si comprende la ragione per cui la rappresentazione del capitalismo, come «unique happening» 85 dovrebbe essere incompatibile con l'individuazione delle differenze tra vari modelli di capitalismo. La rilevanza delle peculiari articolazioni culturali dei sistemi nazionali (indagata successivamente da storici e sociologi dell'economia e oggetto di interessanti riflessioni teoriche tra gli scienziati sociali contemporanei ${ }^{86}$ ) non sminuisce certamente i tratti unitari del capitalismo, esalta semmai la forza unificante dello 'spirito del capitalismo'. La vocazione unitaria à la Sombart rischia, infatti, di portare all'adozione di categorie fondamentalmente destoricizzate e con scarso potere esplicativo nei riguardi dei sistemi storici analizzati. In omaggio alla costruzione di un «quadro unitario» si rischia di rappresentare sistemi storici concreti, «mettendo assieme fatti e cifre del Duecento e Trecento con fatti e cifre di due o tre secoli più tardi $\gg^{87}$ :

voler comprendere entro uno stesso quadro, con gli stessi colori e la stessa luce, la situazione di Venezia, di Genova, di Firenze, di Marsiglia, di Montepellier, di Barcellona, di Ypres, di Bruges, nel Trecento o Quattrocento, con quella della Sassonia e della Prussia, della Francia centrale, dell'Inghilterra e della Scozia negli stessi anni, significa voler forzare la natura dei fatti e darne una rappresentazione completamente diversa dalla realtà ${ }^{88}$.

\section{Convergenze inattese: Gustav Schmoller e Alberto Bertolino interpreti di Sombart}

È interessante rilevare che in Italia, soprattutto ad opera di Bertolino, si sviluppa anche una impegnativa riflessione sul futuro del capitalismo e sul ruolo svolto, al suo interno, dai fattori soggettivi. Su tale aspetto, emerge, piuttosto sorprendentemente, una convergenza tra G. Schmoller (uno dei maestri di Sombart) e Alberto Bertolino, che - in sintonia con buona parte della cultura economica e filosofica italiana - non ha certo espresso giudizi benevoli sulla scuola storica tedesca dell'economia e sul padre della «giovane scuola dell'economia» ${ }^{89}$.

85 I. Wallerstein, From Feudalism to Capitalism: Transition or Transitions, «Social Forces», 55 (2), 1976, p. 277.

86 Come si accennava, il tema delle differenti varietà dei sistemi economici nel capitalismo è attualmente oggetto di studi di grande interesse in ambito economico, sociologico e politologico. Si vedano: P.A. Hall, D. Soskice, Varieties of Capitalism. The Institutional Foundation of Comparative Advantage, Oxford University Press, Oxford 2001; M.P. Arsenault, The Effects of Political Institutions on Varieties of Capitalism, Palgrave Macmillan, Cham 2017.

87 Luzzatto, Dai servi della gleba agli albori del capitalismo, cit., p. 517.

${ }_{88}$ Cfr. ivi, p. 516.

89 A. Bertolino, Werner Sombart e Max Weber nel dissolvimento della scuola storica tedesca del pensiero economico, Id., Scritti e lezioni di storia del pensiero economico, a cura di P. Barucci, Giuffrè, Milano 1979, pp. 495-496. Su questo si veda M. Dardi, Alberto Bertolino attraverso il fascismo, in P. Barucci, P. Bini, L. Conigliello (a cura di), Economia e Diritto in Italia durante il Fascismo, University Press, Firenze 2017, p. 8. 
Per quanto riguarda Schmoller, farò riferimento alla recensione di Der moderne Kapitalismus del 1903 (Schmoller, dopo quella data, non esprime più giudizi di ampio respiro sull'opera sombartiana; muore nel 1917); per quanto riguarda Bertolino utilizzerò i seguenti saggi: L'opera maggiore di Werner Sombart; Il testamento politico economico di Werner Sombart; Werner Sombarte Max Weber nel dissolvimento della scuola storica tedesca del pensiero economico e L'opera di Werner Sombart nel centenario della nascita.

La recensione di Schmoller segnala la rilevanza dell'opera di Sombart, ma anche la persistenza di problemi che, risolti solo nominalmente, si trasformano in limiti significativi nell'analisi della società moderna. Schmoller, sottolineando la stretta relazione tra Der moderne Kapitalismus di Sombart e Das Kapital di K. Marx, rileva che Sombart già nel titolo dell'opera enfatizza, marxianamente, il ruolo dei «processi economici tecnico-meccanici» come «causa unica della condizione economica e sociale». Viene, cioè, ignorato il ruolo di «tutte le cause psicologiche, etiche e istituzionali $\gg^{90}$, al di là delle critiche sombartiane al materialismo storico. Infatti, chiamando «capitalismo» l'insieme dei fenomeni costitutivi della società moderna, li si rappresenta come se fossero «una conseguenza del Capitale per sé e non di fattori psichici da lui stesso definiti come la causa diretta» (《als ob es eine Folge des Kapitals an sich und nicht der von him selbst als direkte nähere Ursache geschilderten psychischen Faktoren wäre») ${ }^{91}$. D'altra parte, proprio Sombart aveva esplicitamente sottolineato, che nell'analisi della genesi del capitalismo sono completamente fuori luogo i riferimenti alla «natura umana e alle sue interne forze motrici» ( «Hinweise auf die menschliche Natur und ihre innewohnende Triebe sind völlig deplaciert» $)^{92}$. In tal modo, però, il riferimento ai «fattori soggettivi» diventa una ricorrente petizione di principio, che, sul piano analitico, assume una funzione meramente sussidiaria, rinviando - surrettiziamente - alla «causa ultima» (di natura meramente economica) del mutamento sociale ${ }^{93}$. I fattori soggettivi da forze motrici diventano elementi di trasmissione e, addirittura, di amplificazione di tendenze che, in ogni caso, si sarebbero realizzate a causa della forza dei meccanismi endogeni del sistema economico e della sua evoluzione 'tecnico-meccanica'.

Anche Bertolino enfatizza l'importanza dell'opera di Sombart, mettendo in guardia contro $i$ «pregiudizi $\gg$ che ne hanno caratterizzato l'interpretazione e insistendo sul fatto che i limiti di essa vanno messi a fuoco «con un' indagine interna al suo sistema di pensiero ${ }^{94}$, a partire dalla duplice finalità che egli persegue:

G. Schmoller, Werner Sombart: Der Moderne Kapitalismus, in H.H. Nau (ed.), Gustav Schmoller. Historisch-ethische Nationalökonomie als Kulturwissenschaft, Metropolis, Marburg 1998 (ed. orig. 1903), p. 211.

92 Sombart, Der moderne Kapitalismus, cit., p. 379.

93 A. Cavalli, Introduzione a Sombart, in Sombart, Il capitalismo moderno, cit., p. 29; si veda anche: V. Gioia, Werner Sombart: il capitalismo moderno e il suo futuro, cit., pp. 20 sgg.

94 Bertolino, Considerazioni critiche sulla metodologia sombartiana, cit., p. 174. 
a) conciliare, nell'analisi del moderno capitalismo, l'approccio teoretico con i risultati delle ricerche empiriche;

b) indagare il ruolo decisivo dei fattori soggettivi contro ogni visione 'tecnologica' e 'deterministica' dell'evoluzione sociale.

Secondo Bertolino, è sbagliato «assimilare il metodo di Sombart con quello "naturalistico"» e positivistico, che egli ha sempre severamente criticato. Sombart tiene fermi, in tutta la sua opera, due postulati fondamentali:

1) la conoscibilità "interiore" della realtà delle azioni umane, per cui lo studioso può interpretare, "comprendere", gli accadimenti della vita sociale in quanto è egli stesso un uomo; 2) la organizzazione sistematica dell'interpretazione di tali azioni, per cui le loro manifestazioni possono essere conosciute se coordinate criticamente in relazione allo spirito della società umana considerata ${ }^{95}$.

I fatti umani non sono «considerati in se stessi», ma in base ai «motivi e [ai] modi per cui e in cui gli uomini li producono; che è il modo per individuare la loro essenza $\gg^{96}$, cogliendone lo spirito, la forma e la tecnologia e inserendole in una «struttura sociologica» che, ricostruita con un «metodo culturale», consenta di «comprendere» «la realtà economica e sociale nella sua totalità» ${ }^{97}$. In Sombart, «le condizioni materiali della vita economica possono considerarsi operative nella formazione e nello sviluppo del sistema capitalistico se ed in quanto vi aliti» lo «spirito del capitalismo» che dà un significato univoco all'insieme dei fatti indagati e ai comportamenti degli attori sociali ${ }^{98}$.

Purtroppo, Sombart non è conseguente né sul piano epistemologico (rapporto tra analisi teorica e analisi empirica), né sul piano dell'indagine dei fattori soggettivi. Secondo Bertolino, l'aspirazione a conciliare teoria e analisi empiri$\mathrm{ca}$ è, nel complesso, deludente. La soverchiante ricchezza di ricerche empiriche e di materiali storiografici (generalmente di seconda mano), che egli cita, non sono utilizzati per definire ipotesi teoriche da sottoporre a ulteriori verifiche, ma a giustificare ipotesi aprioristicamente definite. Ne deriva che: la «raccolta di fatti $\gg$ che avrebbe dovuto eventualmente riorientare la ricerca teorica si trasforma spesso - senza opportune mediazioni - in teoria ${ }^{99}$, producendo generalizzazioni teoriche per via eminentemente deduttiva in base ad assunti iniziali, adottati senza opportune valutazioni critiche. Ne risulta una continua oscillazione tra una «esagerata concezione della teoria» (cosa che rimproverava agli economisti) e una certa subalternità alla «storia» o a quei materiali empirici, che egli isola come decisivi nella spiegazione dei fenomeni analizzati: «intendendo la prima esclusivamente nel senso tradizionale di un'elaborazione fon-

95 Ibidem.

96 Ivi, p. 179.

97 Ivi, p. 177.

98 Ivi, p. 172. Si veda anche: Bertolino, Werner Sombart e Max Weber, cit., p. 528.

99 Bertolino, Considerazioni critiche sulla metodologia sombartiana, cit., p. 178. 
damentalmente deduttivistica delle leggi dell'attività economica, e la seconda come pura descrizione empirica ${ }^{100}$.

Per quanto riguarda l'analisi dei fattori soggettivi, la riflessione sombartiana pare ancora più paradossale. Essa si sviluppa attraverso la ricerca di modelli di soggettività che sembrano aver il solo scopo di assicurare una lettura unitaria dei fenomeni indagati, eludendo la faticosa ricerca sul modo in cui le forme di soggettività, radicate nelle condizioni materiali di esistenza, si manifestano storicamente con idee, atteggiamenti culturali originali e strategie coerenti con i problemi da risolvere. Non è un caso che egli introduca la categoria dei «fondatori», cui attribuisce nuovi modelli di agire economico, come «eletta schiera di antenati spirituali», «assolutamente» «sradicati» «dal punto di vista sociale ${ }^{101}$. Il «fondatore», in tal senso, «proviene da ogni possibile strato sociale, ma quel che lo contraddistingue in maniera del tutto particolare è che egli non porta l'impronta di nessuno degli strati sociali da cui proviene. Egli è nato libero, quasi caduto dal cielo» ${ }^{102}$. Il fondatore è caratterizzato da una radicale alterità ed esogeneità rispetto alle comunità e a questa vocazione a-comunitaria $\mathrm{e}$ anti-comunitaria Sombart attribuisce quella 'forza creativa' che trasforma l'economia e la società. Ora, se la dinamica del capitalismo è determinata da poche variabili astoriche (quasi cadute dal cielo) ed esogene rispetto ai sistemi reali, è evidente che all'attenuarsi degli effetti di quelle variabili sulla società, essa sarà condannata ad un'evoluzione meramente meccanica e ripetitiva ${ }^{103}$ : «non può esserci un progresso nell'andamento della vita sociale ${ }^{104}$.

Non ha torto, dunque, Bertolino quando denuncia in Sombart la «tendenza ad insinuare il mito nell'interpretazione storica $\gg^{105}$, manifestando una «visione mistica e trascendentale dei rapporti sociali, la cui perfezione [...] verrebbe dall'alto e dal di fuori, non dalla volontà ed operosità di coloro che li costituiscono $\gg^{106}$. Infatti, una volta acclarato che la «macchina economica $\gg$ ha ormai una forza intrinseca così rilevante da non poter essere riorientata dalle 'soggettività' - che essa determina - in funzione del perseguimento di finalità sociali, resta il ricorso alla «divinizzazione degli intenti e delle opere di alcuni individui necessariamente preposti alla guida delle collettività umane ${ }^{107}$. Il Führerprinzip obbedisce a questa stessa logica: è, ancora una volta, un espediente esogeno, un deus ex machina, che dovrebbe ridare al 'popolo' le sue valenze comunitarie e il senso del suo essere un 'popolo'. Sombart ritorna al «misticismo

${ }^{100}$ Cfr. ivi, p. 183. Considerazioni analoghe in A. Bertolino, Werner Sombart e Max Weber, cit., pp. 506-507.

${ }^{101}$ Sombart, Il capitalismo moderno, cit., pp. 269-270.

${ }^{102}$ Ibidem; corsivo mio.

${ }^{103}$ Bertolino, Werner Sombart e Max Weber, cit., p. 484.

${ }^{104}$ Bertolino, Considerazioni critiche sulla metodologia sombartiana, cit., p. 180.

${ }^{105}$ Cfr. ivi, p. 180.

${ }^{106}$ Bertolino, Werner Sombarte Max Weber, cit., p. 484. Si veda anche: Iannone, Umano, ancora umano. Per un'analisi dell'opera Sull'uomo di Werner Sombart, cit., p. 57.

${ }^{107}$ Bertolino, Considerazioni critiche sulla metodologia sombartiana, cit., p. 181. 
e [...] romanticismo mülleriano» (che egli aveva aspramente criticato). Insomma, conclude Bertolino, Sombart che nella definizione dei caratteri del «moderno capitalismo» aveva preso l'avvio da una «rappresentazione ideologica [...] dell'epoca medievale», ora ripropone quella stessa visione relativamente ai caratteri del capitalismo contemporaneo, finendo con il diventare antimodernista e anticapitalista ${ }^{108}$.

Schumpeter, nella nota recensione del 1927 (Sombarts Dritter Band) rileva che l'orientamento scientifico complessivo di Der moderne Kapitalismus si pone nel contesto dello Schmollerprogramm ( $\ll$ in der Richtung des Schmollerprogrammes»), cui dà un nuovo impulso ${ }^{109}$. Questo giudizio non mi sembra completamente condivisibile. Certo, come tutti i rappresentanti della scuola storica dell'economia, egli prende l'avvio dalla esigenza di indagare il ruolo dei fattori soggettivi nella dinamica economica e sociale, ma sia sul piano epistemologico (conciliazione tra teoria e indagini empiriche), che sul piano analitico egli finisce con il porsi al di fuori dello Schmollerprogramm, smarrendo il riferimento alla circolarità storica tra condizioni materiali d'esistenza, dimensione culturale e contesti politico-istituzionali che Schmoller poneva al centro del suo programma di ricerca: «Ogni organizzazione economica determinata non ha solo come fine quello di produrre beni, ma anche quello di essere il ricettacolo $(G e f a ̈ \beta)$, la causa produttrice, la matrice di fattori morali senza i quali la società non potrebbe vivere $\gg^{110}$.

Senza il riferimento a tale circolarità, culturalmente e istituzionalmente radicata, appare difficile sia la spiegazione unitaria dei fenomeni sociali, sia la messa a punto di politiche in grado di correggere «l'ingiustizia economica, che si manifesta nella vita sociale attuale». Questa, infatti, «non è esclusivamente un residuo dei tempi passati; [...] una gran parte di questa ingiustizia deriva da ciò che la morale e il diritto non sono in grado di fare in presenza di una situazione economica nuova, lasciando così relativamente più libertà d'azione alla superiorità del più forte dal punto di vista economico, alla dolosa scaltrezza, all'inganno, alla soverchieria $\gg^{111}$. Se, sottolinea Schmoller, l'elemento dinamico è dato solo dalle condizioni della produzione e dalla tecnica, quando queste divengono disumane o, per lo meno, non in grado di far scaturire dai meccanismi endogeni del sistema economico finalità umane, tutto ciò viene meccanicamente ribaltato sull'intera società che assume un carattere disumanizzante, cui non si può porre rimedio: moventi soggettivi, istituzioni e valenze etiche non hanno più alcun ruolo nei processi di mutamento sociale.

Se Schmoller nel 1903 aveva previsto i possibili esiti dell'analisi di Sombart, Bertolino li evidenzia con riferimento alla lettura dell'intera sua opera: senza

${ }^{108}$ Bertolino, Werner Sombart e Max Weber, ivi, p. 479. Si veda anche: H. Jeffrey, Reactionary Modernism, Cambridge University Press, Cambridge 1984, in particolare pp. 130-151.

109 J.A. Schumpeter, Sombarts Dritte Band, «Schmollers Jahrbuch», 51, 1927, p. 352.

${ }^{110}$ Schmoller, Teorie vaganti e verità stabili nel campo della ricerca sociale, cit., p. 54.

${ }^{111}$ Cfr. ivi, pp. 115-116. 
soggettività, storicamente radicate, il sistema economico viene caratterizzato come sequenza meccanica di stadi determinati da progressiva degenerazione, poiché li si compara con sistemi di relazioni sociali astorici e mistici. Il disincanto dell'analista sociale nei confronti della durezza e asprezza degli eventi storici si trasforma in delusione e nella riproposizione di modelli premoderni, a forte densità ideologica. Epstein scriveva che $\ll$ Sombart was an artist as well as a scholar, gifted with imagination in a high degree. His artistic temperament sometimes led him to exaggerated judgments or over-statements, or even contradictions. But he had great learning and an attractive style, so that his books read like romances $\gg^{112}$. Più recentemente Geminello Alvi, enfatizzando il ruolo essenziale dell'immaginazione scientifica nell'analisi della realtà, ha attribuito a Sombart la costruzione di «una scienza per immagini», che, considerando la scienza economica come Geisteswissenschaft, non ritiene che essa sia confinabile alla «esclusiva collezione del materiale empirico o della costruzione di modelli meccanici ${ }^{113}$. Non sottovaluto né la forza di questa argomentazione, né il ruolo dell'intuizione e dell'immaginazione scientifica, poiché - come ampiamente dimostrato - ad esse, e non alla meccanica applicazione delle regole formali, sono legate le innovazioni epistemologiche ${ }^{114}$. Tuttavia, resta vero che le narrazioni e le grandi intuizioni devono poi tradursi in modelli di spiegazioni scientifiche razionalmente accettabili. Se ci si riferisce ai rappresentanti della GHS, tale tema è stato affrontato in maniera esplicito e - a mio parere - in modo più conseguente rispetto allo Schmollerprogramm, da Arthur Spiethoff, che l'ha tradotto nell'ipotesi di una «anschauliche Theorie»o «geschichtliche-anschauliche Theorie $\gg$. Il senso di tale approccio è difficile da cogliere immediatamente al di fuori dei paesi di lingua tedesca, per la difficoltà di tradurre «anschauliche»e «Anschaulichkeit» e per una diffusione inadeguata dei testi epistemologici di Spiethoff ${ }^{115}$. Io ho tradotto con 'teoria storico-concreta', recuperando anche la definizione spiethoffiana di Realtype che egli elabora per differenziare la sua analisi da quella dall'idealtipo weberiano ${ }^{116}$. L'intento di Spiethoff era duplice:

112 M. Epstein, Obituary, «The Economic Journal», 51 (204), 1941, p. 526.

113 G. Alvi, Le seduzioni economiche di Faust, Adelphi, Milano 2014, p. 27.

114 T.S. Kuhn, La struttura delle rivoluzioni scientifiche, Einaudi, Torino 1978, spec. pp. 139 sgg. e T.S. Kuhn, La tensione essenziale, Einaudi, Torino 1985, spec. pp. 115 sgg. Si veda anche: J. Bronowski, Science and Human Values, Harper \& Row Publishers, New York 1965, pp. 12 sgg.

115 Solo nell'economia, sottolineava amaramente Schumpeter - proprio con riferimento all'analisi di Spiethoff, «la barriera linguistica» gioca un ruolo così rilevante da portare a trascurare contributi importanti per lo sviluppo della scienza. E questo, aggiungeva, «illustra uno stato di cose che è molto generale e spiega molto nella storia dell'economia» (J.A. Schumpeter, Storia dell'analisi economica, vol. III, Bollati Boringhieri, Torino 1990, p. 1383).

116 A. Spiethoff, Die allgemeine Volkswirtschaftslehre als geschichtliche Theorie. Die Wirtschaftsstil, «Schmollers Jahrbuch», 56, 1932, pp. 51-84; A. Spiethoff, Anschauliche und reine volkswirtschaftlich Theorie und ihre Verhältnis zueinander, in Synopsis. Festschrift für Alfred Weber, Schneider, Heidelberg 1948, pp. 569-664. Su questo si veda: V. Gioia, Historical Changes and Economics in Arthur Spiethoff's Theory of Wirtschaftsstil (Style of an Economic System), in Koslowski, Methodology of the Social Sciences, cit., pp. 168-190. 
enfatizzare il ruolo dell' intuizione nell'analisi della realtà, specialmente con riferimento alle scienze sociali e, in secondo luogo - prendendo le distanze anche da Sombart e dalla sua definizione di 'sistema economico'117 - porre l'accento sul fatto che, dati gli oggetti di ricerca, i meccanismi esplicativi hanno una loro specifica autonomia, che deve svilupparsi in contesti logici coerenti con $\mathrm{i}$ «programmi di ricerca» delineati, orientando a tal fine la ricerca empirica. Questo, secondo il suo punto di vista, consente di enfatizzare la peculiarità degli «oggetti storici» tipici delle scienze sociali e, nello stesso tempo, di non sottovalutare la «funzione euristica» di approcci, come quello dell'economia pura, le cui spiegazioni scientifiche prescindono dalla storicità dei fenomeni indagati, in virtù dell'uso di un impianto esclusivamente deduttivo ${ }^{118}$. In tal modo, egli aveva tentato di superare le artificiose contrapposizioni del Methodenstreit, rilevando che il discrimine tra economia storica ed economia pura non andava cercato nell'applicazione delle regole logiche (da utilizzare con lo stesso rigore in ogni ambito esplicativo), bensì nella definizione di programmi di ricerca che sono da ritenere egualmente legittimi, in funzione delle diverse configurazioni degli oggetti di ricerca e dei «compiti specifici» che essi pongono ${ }^{119}$. Il fatto è che proprio su questo piano, che continua a porre questioni significative per lo sviluppo attuale delle scienze sociali ${ }^{120}$, Sombart si era mosso in maniera inadeguata e contraddittoria ${ }^{121}$.

\section{Conclusioni}

Francamente, ritengo oziosol'interrogativo se sia ancora utile leggere l'opera sombartiana. Sombart è un classico delle scienze sociali, che ha avuto meriti indubbi sia nell'apertura di nuovi ambiti per la ricerca economico-sociologica, sia nella sua riconosciuta capacità di rendere 'accademicamente' fruibili programmi di ricerca (a cominciare da quello marxiano) che, ai suoi tempi, si ponevano ai margini della riflessione scientifica, in quanto considerati solo oggetto di dibattito politico. Tutto questo è stato realizzato attraverso un coraggioso discorso epistemologico, teso a superare i limiti del Methodenstreit, con l'intento di conciliare analisi teorica e indagini empiriche, nel tentativo di cogliere la dimensione storica degli oggetti di ricerca e le cause del mutamento sociale. Non ritorno sulle tante contraddizioni di Sombart, ma considero un errore utilizzare i limiti del suo discorso scientifico per sottolineare l'irrilevanza delle questio-

${ }^{117}$ Cfr. A. Spiethoff, Anschauliche und reine volkswirtschaftlich Theorie, cit., pp. 577 sgg.

118 A. Spiethoff, Business cycles, «International Economic Papers», 3, 1953, p. 75.

119 A. Spiethoff, The "Historical" Character of Economic Theories, «The Journal of Economic History», 12 (2), p. 135.

${ }^{120} \mathrm{Su}$ questo si veda: I. Wallerstein, The Limits of Nineteenth Paradigm. Unthinking Social Science, Temple University Press, Philadelphia 2001, pp. 227 sgg.

${ }^{121}$ Si veda: Geleitwort zur zweiten Auflage, in W. Sombart, Der moderne Kapitalismus, cit., specialmente pp. XVI sgg. Si veda anche: W. Sombart, Die Drei Nationalökonomien, Verlag von Duncker \& Humblot, München und Leipzig 1930. 
ni che egli pose. La consapevolezza della difficoltà dell'impresa non può - sic et simpliciter - essere considerata come prova dell'impossibilità di pervenire a soluzioni soddisfacenti sul piano epistemologico e analitico ${ }^{122}$. In fondo, come proprio Sombart rilevava, «anche se la visione dello scienziato non è corretta, egli può contribuire all'avanzamento della scienza $\gg^{123}$, a condizione che la sua analisi avvii percorsi, che consentano confronti razionali con le tante unsettled questions presenti nelle scienze sociali contemporanee. Tali interrogativi sono generalmente ignorati o considerati con superficialità non perché irrilevanti, ma in quanto incompatibili con l'orientamento mainstream. Tuttavia, è certamente vero che molte delle questioni che egli pose continuano ad essere oggetto di interessanti dibattiti nelle scienze sociali contemporanee, con lo scopo di far emergere i limiti dell'approccio mainstream (soprattutto in economia) e la necessità di individuare percorsi che raccordino in modo più soddisfacente teorie e ricerca empirica, emancipandoci da quella $\left\langle\right.$ cultural hybris ${ }^{124}$, che considera irreversibile la divaricazione tra il progressivo perfezionamento delle tecniche analitiche e la insoddisfacente rilevanza empirica delle spiegazioni scientifiche: «What was previously regarded as standard economic theory seems to many to have failed us, not only in addressing the series of crises which began in 2007, but also in being implicated in the causes of the crises $\gg^{125}$. Sono, come abbiamo visto, interrogativi che, in un diverso contesto storico e scientifico, aveva posto W. Sombart ${ }^{126}$.

Inoltre, sul piano dei contenuti non può essere sottovalutata la portata attuale di alcuni temi che Sombart aveva individuato come centrali nell'evoluzione del capitalismo dei suoi tempi: democrazia e mercato, capitalismo e socialismo, i caratteri del moderno individualismo. Non possiamo, in queste brevi conclusioni, sviluppare l'analisi di questi aspetti, ma è indubbio che l'evoluzione del capita-

${ }^{122}$ Su tale aspetto, si vedano: G.M. Hodgson, How Economics Forgot History, Routledge, London-New York 2001, spec. pp. 3-40; B. Schefold, Geschichte der Wirtschaftstheorie und Wirtschaftsgeschicte: Einleitung, «Jarbuch für Wirtschaftsgeschichte», 50 (1), 2009, pp. 9-26; E.J. Nell, On the History of Economic Theory and the Emergence of Capitalism, «Jarbuch für Wirtschaftsgeschichte», 2009, 1, pp. 103-134.

${ }^{123}$ W. Sombart, Problemen des deutschen Wirtschaftsleben. Erstrebtes und Erreichtes, Walter de Gruyter \& co., Berlin-Leipzig 1937, p. 763 (traduzione mia).

124 R.N. Anshen, Convergence, in A. Lőwe, Has Freedom a Future?, Praeger Publisher, New York 1988, p. XIV.

${ }^{125}$ S.C. Dow, Foundation for New Economic Thinking. A Collection of Essays, Palgrave MacMillan, New York 2012, p. 1. Si veda anche, nello stesso volume, il capitolo: The Non-neutrality of Formalism, pp. 140-160.

${ }^{126}$ Su questo si vedano: G. Chaloupek, Long Term Economic Trends in the Light of Werner Sombart's Concept of Spätkapitalismus, in Backhaus (ed.), Werner Sombart, cit., vol. II, p. 177; B. vom Brocke, Werner Sombart 1863-1941. Capitalism-Socialism, in Backhaus (ed.), Werner Sombart, cit., vol. I, p. 102; W. Mitchell, Human Behavior and Economics. A Survey of Recent Literature, «The Quarterly Journal of Economics», 29 (1), 1914, p. 36; V. Gioia, Capitalism and Judaism in Werner Sombart: a Contribution to the Analysis of Capitalist Rationality and its Limits, cit., pp. 15-38. 
lismo contemporaneo ha esasperato le criticità individuate da Sombart. La forza della 'macchina economica' ha eroso progressivamente gli spazi decisionali delle istituzioni democratiche, avallando l'idea di una technological inevitability dell'evoluzione sociale, secondo principi che tendono a 'emancipare' le politiche economiche e sociali (e questo viene considerato un segno di efficienza del sistema) da moventi morali, politici e ideali. Viene enfatizzata l'idea che ci muoviamo in «a fundamentally unchanging social universe ${ }^{127}$, che esclude cambiamenti strutturali del tipo di quelli che si erano verificati nella transizione dalle società premoderne al capitalismo. In base a questa logica, e all'analisi di quanto si era realizzato dopo la Rivoluzione d'ottobre nell'Unione Sovietica, appare comprensibile il giudizio di Sombart sulla indistinguibilità tra Spätkapitalismus e socialismo. Il socialismo non è stato in grado di introdurre forme di orientamento etico nella vita sociale e, di conseguenza, non è stato in grado di ridare un'anima al sistema economico che ha ereditato dal capitalismo. Esso ha sviluppato ulteriormente quei processi di razionalizzazione imposti dal sistema economico, che - sul piano politico e sociale - si sono tradotti in una severa riduzione delle libertà di scelta degli individui e dei popoli. Si tratta di una conclusione che, espressa in maniera provocatoria da Sombart, era ampiamente diffusa ai suoi tempi e fondamentalmente condivisa - tra gli altri - da M. Weber e Schumpeter ${ }^{128}$.

Di particolare interesse, può essere anche l'analisi dei limiti della riflessione sombartiana sui caratteri del moderno individualismo. Sombart, come altri rappresentanti della GHS, intende dimostrare la rilevanza dei fattori soggettivi nel mutamento sociale, tuttavia nella sua riflessione tali fattori, che in origine erano oggetto di indagine teoretica nel contesto di un peculiare 'tipo storico' di società (il capitalismo europeo), finiscono con l'assumere un ruolo analiticamente marginale, come evidenziato da Bertolino. Di conseguenza, Sombart - messi a fuoco i meccanismi di funzionamento del 'sistema economico' e le sue fondamentali relazioni causali - regredisce verso quella visione dei soggetti storici che, da giovane, aveva aspramente criticato, ricadendo in un approccio tipico, per dirla con Marx, del 'materialismo volgare'. Secondo il suo punto di vista, il capitalismo ha creato un ambiente economico e sociale che ha irreversibilmente modificato la 'natura umana', al punto da rendere poco plausibile l'ipotesi di un mutamento strutturale del sistema determinato da originali forme di attivismo dei soggetti.

Se si considera la letteratura sociologica e filosofica contemporanea, si è colpiti dall'ampia diffusione di questa concezione sombartiana e se ne può anche

${ }^{127}$ I. Wallerstein, Does Capitalism have a Future?, Oxford University Press, Oxford 2013, p. 5.

${ }^{128}$ M. Weber, Economia e società, Edizioni di Comunità, Milano 1968, pp. 203 sgg.; J.A. Schumpeter, Capitalism, Socialism and Democracy, Harper Colophon Edition, New York 2008, p. 206. Su questo si veda: V. Gioia, Economics and Sociology meet Socialism: Sombart, Durkheim and Pareto, in R. Soliani (ed.), Economic Thought and Institutional Change in France and Italy, 1789-1914, Springer, Berlin 2017, spec. pp. 210 sgg.; M. Recalcati, L'uomo senza inconscio, Raffaello Cortina, Milano 2010, in particolare pp. 3-26; C. Lash, L'Io minimo, Neri Pozza, Vicenza 2018; C. Taylor, Sources of the Self. The Making of Modern Identity, Harvard University Press, Cambridge, Massachusetts 1989. 
cogliere l'errore analitico che l'ha resa possibile. Di norma, si prende l'avvio dall'analisi di un tratto predominante del comportamento individuale, riscontrato in una fase di sviluppo della società contemporanea, definendolo come un dato costitutivo generale della 'natura umana'. A partire da esso, l'individuo può essere così rappresentato come agente edonistico, narcisista, consumista, senza inconscio, rapace nei confronti dell'ambiente naturale e sociale; insomma come un attore completamente e irreversibilmente determinato «by the forces of the environment $\gg^{129}$. Questo approccio sacrifica, però, quella dotazione della 'natura umana' che ha determinato in passato (e continuerà a determinare in futuro) cambiamenti nell'economia e nella società: la creatività. Tale creatività ha certamente un fondamento storico, poiché i modelli di soggettività sono determinati dai contesti in cui gli uomini vivono, ma è la dotazione umana che consente di superare quelle stesse condizioni iniziali, quando gli individui siano sollecitati a progettare originali soluzioni, per rispondere alle nuove sfide poste dall'evoluzione sociale $\mathrm{e}^{130}$. Appare singolare che la categoria di creatività umana venga considerata inefficace ai fini dell' individuazione di nuove finalità generali funzionali alla riorganizzazione dell'agire economico e sociale, e sia confinata esclusivamente all'analisi dei suoi effetti su progresso scientifico e scoperta di espedienti tecnologici funzionali all'incremento della ricchezza materiale. Viene, cioè, trascurato il fatto che la creatività umana, in condizioni storicamente determinate $\mathrm{e}$ in virtù delle trasformazioni culturali degli agenti sociali, possa articolarsi (come affermava il Sombart di Sozialismus und soziale Bewegung im 19. Jahrhundert) in originali visioni strategiche, finalizzate alla costruzione di modelli di organizzazione sociale, in grado di avviare a soluzione i tanti problemi posti dall'evoluzione del mondo contemporaneo ${ }^{131}$.

${ }^{129}$ Sulla diffusione di tale approccio si vedano: F.A. Hayek, Individualism: True and False, Dublin and Oxford 1946, repr. in Id., Individualism and Economic Order, London 1949.

${ }^{130} \mathrm{Su}$ questo si veda l'esemplare contributo di J. Dewey, Human Nature and Conduct. An Introduction to Social Psychology, Random House, New York 1922.

${ }^{131}$ Si veda: J. Dewey, Individualism Old and New, Prometheus Book, Amherst, NY 1984, specialmente pp. 71-83. 